Artículo

\title{
Tamaño de la muestra para estimar el promedio de variables agronómicos en yuca
}

\author{
André Schoffel ${ }^{1}$ \\ Sidinei José Lopes ${ }^{1}$ \\ Jana Koefender ${ }^{2}$ \\ Alessandro Dal'Col Lúcio' ${ }^{1}$ \\ Juliane Nicolodi Camera ${ }^{2}$ \\ Diego Pascoal Golle ${ }^{2}$
}

${ }^{1}$ Universidad Federal de Santa María. AV. Roraima núm. 1000, Santa Maria, Rio Grande do Sul, Brasil. (andre-schoffel@hotmail.com; sjlopes2008@gmail.com; adlucio@ufsm.br). ${ }^{2}$ Universidad de Cruz Alta. Carretera municipal Jacob Della Méa km 5.6, Parada Benito, Cruz Alta, Rio Grande do Sul, Brasil. (jkoefender@unicruz.edu.br; ju_camera@yahoo.com.br; dgolle@unicruz.edu.br).

§Autor para correspondencia: andre-schoffel@hotmail.com.

\section{Resumen}

En experimentos agrícolas, medir todas las plantas que constituyen una unidad experimental es el método más apropiado para estimar promedios. Este estudio analizó tamaños de muestra (número de plantas) para estimar promedios de caracteres agronómicos en yuca. El experimento para la producción de plántulas se realizó en cuatro fechas de siembra: 22 de septiembre, 7 de octubre, 19 de octubre y 25 de noviembre de 2017. Los caracteres evaluados fueron la altura de la planta en la siembra, el número de hojas en la siembra, el número de hojas a los 7 días después de la siembra, el número de hojas en el trasplante y la altura de la planta en el trasplante. Las plántulas se trasplantaron al campo el 9 de noviembre, 24 de noviembre, 3 de diciembre y 26 de diciembre. Las características de la raíz y el rendimiento de la raíz se midieron en la cosecha. Se midieron las siguientes estadísticas: mínimo, máximo, rango, media, mediana, varianza, desviación estándar y coeficiente de variación. El tamaño de la muestra se determinó mediante remuestreo con reemplazo cuando el rango del intervalo de confianza (IC) de 95\% fue de 10\%, 20\%, 30\% y 40\% de la estimación promedio. Se necesitaron tamaños de muestra más grandes para estimar los promedios de rasgos con un rango de IC de $95 \%$ de $10 \%$ de la estimación promedio; es decir, cuando la precisión de la estimación fue mayor. En contraste, se requirieron tamaños de muestra bajos para estimar promedios de los caracteres en los tiempos de siembra con un rango de IC de 95\% de 40\%. Para medir la producción de plántulas y rendimiento de tallos y raíces, 63 y 153 plantas son suficientes para estimar promedios cuando el rango del intervalo de confianza de $95 \%$ fue de $20 \%$.

Palabras clave: Manihot esculenta Crantz, diseño experimental, método de multiplicación rápida, propagación vegetativa, tamaño de muestra.

Recibido: enero de 2021

Aceptado: marzo de 2021 


\section{Introducción}

La yuca pertenece a la familia Euphorbiaceae y es una importante fuente vegetal para producir raíces almidonadas, que contribuyen a la seguridad alimentaria en muchos países. Las plantas de yuca están altamente adaptadas a diferentes condiciones de cultivo y constituyen la tercera fuente más importante de energía alimentaria en los trópicos (Hasibuan y Nazir, 2017). Además, las plantas pueden crecer en suelos química y físicamente degradados y en condiciones de estrés hídrico (Egesi et al., 2007).

Aproximadamente $87 \%$ de la producción de yuca en Brasil proviene de granjas familiares, y el rendimiento promedio en este tipo de propiedad es de $5770 \mathrm{~kg} \mathrm{ha}^{-1}$ (Brasil, 2009). Además de las limitaciones en el área de cultivo y la baja inversión tecnológica, los materiales de propagación de baja calidad fisiológica y sanitaria se utilizan en cosechas secuenciales, lo que aumenta el riesgo de enfermedades que impactan significativamente en el desarrollo y rendimiento de las plantas (Silva et al., 2013). Por lo tanto, es crucial implementar y mejorar las técnicas de manejo y de cultivo que permitan la utilización óptima de materiales de propagación de alta calidad.

El método de multiplicación rápida desarrollado por el Centro Internacional de Agricultura Tropical (CIAT) consiste en plantar esquejes con dos o tres cogollos y tallos de cosecha, que están arraigados en macetas con agua y más tarde se utilizan para producir plántulas de yuca en recipientes con sustrato. Este método permite mejor uso de tallos, así como la obtención y selección de plantas con calidad fisiológica y sanitaria, especialmente plántulas sin enfermedades diseminadas por la propagación. Yuguda et al. (2013) han demostrado que el conocimiento limitado sobre las técnicas de manejo es responsable de la baja productividad de la yuca.

Para el trasplante de plantas de yuca a campos de producción, los rasgos de crecimiento y desarrollo de las plantas deben evaluarse con precisión durante el período de producción. Además, la medición de los caracteres de la raíz es crucial, especialmente la capacidad de enraizamiento, que dicta la calidad de la plántula. Sin embargo, la información técnica sobre el crecimiento, desarrollo y rendimiento de tallos y raíces de yuca utilizando el método de multiplicación rápida es escasa. Santos et al. (2009) han demostrado que plantar en la primera estación del año es una alternativa para producir tallos porque las raíces tienen un tamaño pequeño y una forma desigual.

En los experimentos agrícolas, medir todas las plantas que constituyen una unidad experimental es el método más adecuado para estimar los promedios. Sin embargo, dado el tiempo limitado, los recursos financieros y laborales, una población determinada se representa mediante el muestreo. En estos casos, es necesario estimar y utilizar el tamaño de muestra adecuado para aumentar la representatividad de la muestra (Storck et al., 2011). Los intervalos de confianza determinados por el remuestreo se utilizan para calcular el tamaño de la muestra. Esta técnica es independiente de la distribución de probabilidad de los datos y se ha utilizado para estimar los promedios de rasgos en lupino blanco (Burin et al., 2014).

El tamaño de la muestra no siempre se elige de acuerdo con la variabilidad de los datos y el nivel deseado de precisión de la estimación, lo que puede comprometer la calidad del estudio. Schoffel et al. (2020) determinó el tamaño de la muestra para estimar los promedios de rasgos agronómicos en plántulas de yuca procedentes de esquejes de tallos cortados con un número diferente de hojas y diferentes diámetros. 
A pesar de la existencia de adaptaciones en la aplicación del método de multiplicación rápida, pocos estudios han investigado definiciones relevantes, como el tamaño de la muestra y diseños experimentales para el cultivo de yuca. El objetivo de este estudio es determinar el tamaño de la muestra (número de plantas) para estimar los promedios de rasgos en la producción de plántulas, tallos y raíces de plántulas de yuca trasplantadas en cuatro fechas diferentes.

\section{Material y métodos}

Las plántulas de yuca del cultivo Apronta Mesa se plantaron en cuatro fechas de siembra en 2017 (22 de septiembre, 7 de octubre, 19 de octubre y 25 de noviembre) en un modelo de invernadero Van der Hoeven con un sistema automático de riego por niebla, con una profundidad total de riego de aproximadamente $6 \mathrm{~mm} \mathrm{día}^{-1}$ a una temperatura promedio de $25^{\circ} \mathrm{C}$. En cada fecha de siembra, se plantaron y recogieron 300 esquejes cuando contenían de tres a nueve hojas visibles. Las hojas fueron visibles cuando los bordes de uno de los lóbulos no se tocaron entre sí (Schons et al., 2007).

Los esquejes germinados se plantaron en bandejas de plástico negro de 15 celdas con las siguientes dimensiones: longitud, $34 \mathrm{~cm}$; ancho, $21 \mathrm{~cm}$ y altura, $7.8 \mathrm{~cm}$. La dimensión de cada celda era de $6.2 \mathrm{~cm}$ (diámetro superior), $5 \mathrm{~cm}$ (diámetro inferior) y $7.8 \mathrm{~cm}$ (altura), y cada celda tenía cinco agujeros de $6 \mathrm{~mm}$ en la base para el drenaje de agua. Las bandejas estaban previamente llenas con sustrato comercial con riego Mec Plant ${ }^{\circledR}$ para prevenir la deshidratación tisular. Después de eso, se plantaron los esquejes germinados (uno por célula) en surcos de $1 \mathrm{~cm}$ de profundidad por la mañana a una temperatura máxima de $25^{\circ} \mathrm{C}$ para minimizar la deshidratación tisular.

En el momento de la plantación, se midió la altura de la planta (PHP) (desde la base hasta la última hoja visible) y el número de hojas en la plantación (NLP). A los 7 días después de la siembra (DAP), el número de hojas visibles (NL7DAP) se contabilizó de nuevo. A los 30 DAP, las plántulas fueron retiradas del invernadero y transferidas a un criadero para aclimatación durante un mínimo de 5 días, excepto el 25 de noviembre, cuando las plántulas fueron aclimatadas a 23 DAP. El número de hojas en trasplante (NLT) y la altura de las plantas en trasplante (PHT) se midieron en cada fecha de siembra (22 de septiembre, 7 de octubre, 19 de octubre y 25 de noviembre) en 48 , 47, 44 y 31 DAP, respectivamente.

La desecación del cultivo de invierno formada por los cultivos intercalados de cubierta (Avena strigosa Schreb. + Raphanus sativus L.) se llevó a cabo utilizando el herbicida glifosato (3 L ha ${ }^{-1}$ ). La labranza tradicional se realizó por arado. El trasplante de plántulas se realizó el 9 de noviembre, el 24 de noviembre, el 3 de diciembre y el 26 de diciembre (240 plántulas por fecha) en surcos sin fertilizar de $15 \mathrm{~cm}$ de profundidad.

La fertilidad del suelo en el área experimental se analizó en una muestra recogida a una profundidad de 0-20 cm. La muestra presentaba las siguientes características: $\mathrm{pH}$ en agua 5.5; arcilla, 50\%; materia orgánica, 3.3\%; fósforo, $26.2 \mathrm{mg} \mathrm{dm}^{-3}$; potasio, $96 \mathrm{mg} \mathrm{dm}{ }^{-3}$; calcio, $5.9 \mathrm{cmol} \mathrm{dm}^{-3}$; magnesio, $2.4 \mathrm{cmol} \mathrm{dm}^{-3}$; aluminio, $0 \mathrm{cmol} \mathrm{dm}^{-3}$, y saturación base, $68.7 \%$.

La fertilización se llevó a cabo de acuerdo con la recomendación de la Comisión de Química y Fertilidad del Suelo (2016) para el cultivo de yuca. La fertilización del apósito superior se realizó durante el trasplante utilizando $334 \mathrm{~kg} \mathrm{ha}^{-1}$ de fertilizante mineral (5-20-20). La fertilización con 
nitrógeno urea (46-00-00) (70 $\mathrm{kg} \mathrm{ha}^{-1}$ ) se llevó a cabo a los 15 días después del trasplante o al comienzo de la acumulación de almidón. En cultivo de yuca, esto último ocurre cuando las plantas presentan 21 hojas visibles en el tallo principal (Schons et al., 2007).

Las malezas se controlaron en forma manual semanalmente hasta el cierre completo de las filas (hasta tres meses después del trasplante). A falta de lluvias, el riego por aspersión se realizó diariamente hasta el día 15 después del trasplante. El suministro de agua es esencial para producir plántulas de yuca mediante el método de multiplicación rápida.

Las plantas fueron cosechadas el 30 de mayo y la duración del ciclo de crecimiento en cada tiempo de trasplante (9 de noviembre, 24 de noviembre, 3 de diciembre y 26 de diciembre) fue de 203, 188, 179 y 156 días, respectivamente. Otros rasgos medidos fueron la altura de ramificación del tallo principal (BHMS) $(\mathrm{cm})$, número de ramas $(\mathrm{NB})$, número de brotes por tallo (NBS), longitud del tallo (SL) $(\mathrm{cm})$, diámetro del tallo $(\mathrm{SD})(\mathrm{mm})$, peso fresco de la raíz (RFW) ( $\mathrm{g} \mathrm{planta}^{-1}$ ), longitud de la raíz $(\mathrm{RL})(\mathrm{cm})$, diámetro de la raíz $(\mathrm{RD})(\mathrm{mm})$ y el número de raíces $(\mathrm{NR})$.

Los parámetros de diámetro se midieron utilizando una pinza digital. SD se calculó por la media del diámetro basal, intermedio y superior y RD se midió en el tercio superior de las raíces. Las raíces fueron pesadas usando una balanza digital con una resolución de 0.01 gramos. Se analizaron las siguientes estadísticas: mínimo, máximo, rango, media, mediana, varianza, desviación estándar y coeficiente de variación (CV). Las medias se compararon utilizando una prueba $t$ para muestras independientes a un nivel de significancia de $5 \%(p<0.05)$.

Un total de 264, 288, 264 y 244 plantas se midieron en las fechas de siembra 22 de septiembre, 7 de octubre, 19 de octubre y 25 de noviembre, respectivamente, para estimar NLP, NL7DAP, PHP, PHT y NLT. Un total de 213, 231, 232 y 179 plantas se midieron en las fechas de trasplante 9 de noviembre, 24 de noviembre, 3 de diciembre y 26 de diciembre, respectivamente, para estimar BHMS, NB, NBS, SL, SD, RFW, RL, RD y NR.

Sobre la base de estos datos, se planificaron 999 tamaños de muestra, con un tamaño inicial de muestra de dos plantas, y los otros tamaños de muestra se obtuvieron añadiendo secuencialmente una planta hasta un tamaño máximo de 1000 plantas. Después de eso, se realizó remuestreo iterativo para cada tamaño de muestra utilizando 2000 remuestreos con reemplazo. Por lo tanto, se obtuvieron 2000 promedios de rasgos para cada tamaño de muestra. Las siguientes estadísticas se analizaron en base a datos promedios: valor mínimo, percentil del $2.5 \%$, media, percentil de $97.5 \%$ y valor máximo. El rango del intervalo de confianza de $95 \%$ se calculó por la diferencia entre $97.5 \%$ y $2.5 \%$ percentiles.

El tamaño de la muestra para estimar los promedios se calculó por el número de plantas, de las cuales el rango del CI de 95\% fue de 10\%, 20\%, 30\% y 40\% de la estimación promedio. Los análisis estadísticos se realizaron utilizando el software R (R Development Core Team, 2014).

\section{Resultados y discusión}

Hubo diferencias significativas en los promedios de rasgos entre las cuatro fechas de siembra (Cuadro 1). La NLP del 7 de octubre fue inferior a la de las otras fechas de siembra, sin diferencias significativas. Muchas hojas se cayeron de los esquejes plantados el 7 de octubre, lo que puede ser 
confirmado por el NL7DAP promedio. El NL7DAP fue mayor en los esquejes plantados el 25 de noviembre, posiblemente debido a las condiciones ambientales, especialmente la temperatura, lo que favoreció el desarrollo de plántulas en esta fecha de siembra. Además, algunas hojas se cayeron de los esquejes plantados el 25 de noviembre.

Tabla 1. Estadísticas descriptivas sobre el número de hojas en la siembra (NLP), el número de hojas a los 7 días después de la siembra (NL7DAP), la altura de la planta en la plantación (PHP) (cm), la altura de la planta en el trasplante $($ PHT) $(\mathrm{cm})$ y el número de hojas en el trasplante (NLT) de plántulas de yuca cultivadas el 22 de septiembre, 7 de octubre, 19 de octubre y 25 de noviembre.

\begin{tabular}{|c|c|c|c|c|c|}
\hline Estadística & NLP & NL7DAP & PHP & PHT & NLT \\
\hline \multicolumn{6}{|c|}{22 de septiembre } \\
\hline Mínimo & 3 & 1 & 0.4 & 3.3 & 3 \\
\hline Máximo & 9 & 9 & 6.4 & 19.6 & 13 \\
\hline Rango & 6 & 8 & 6 & 16.3 & 10 \\
\hline Media & $5.33^{*} \mathrm{a}$ & $4.5 \mathrm{c}$ & $2.68 \mathrm{a}$ & $10.14 \mathrm{a}$ & $8.74 \mathrm{~b}$ \\
\hline Mediana & 5.5 & 4 & 2.6 & 9.35 & 9 \\
\hline Desviación estándar & 1.34 & 1.45 & 1 & 3.76 & 1.58 \\
\hline Coeficiente de variación (\%) & 25.1 & 32.28 & 37.41 & 37.06 & 18.11 \\
\hline \multicolumn{6}{|c|}{7 de octubre } \\
\hline Estadística & NLP & NL7DAP & PHP & PHT & NLT \\
\hline Mínimo & 3 & 1 & 0.4 & 2.8 & 3 \\
\hline Máximo & 8 & 7 & 5.5 & 16.5 & 12 \\
\hline Rango & 5 & 6 & 5.1 & 13.7 & 9 \\
\hline Media & $4.92 \mathrm{~b}$ & $3.91 \mathrm{~d}$ & $2.3 \mathrm{~b}$ & $8.15 \mathrm{~b}$ & $8.16 \mathrm{c}$ \\
\hline Mediana & 5.5 & 4 & 2.2 & 7.60 & 8 \\
\hline Desviación estándar & 1.42 & 1.31 & 0.86 & 3.05 & 1.56 \\
\hline Coeficiente de variación (\%) & 28.87 & 33.61 & 37.34 & 37.5 & 19.14 \\
\hline \multicolumn{6}{|c|}{19 de octubre } \\
\hline Estadística & NLP & NL7DAP & PHP & PHT & NLT \\
\hline Mínimo & 3 & 2 & 0.6 & 2.4 & 4 \\
\hline Máximo & 8 & 7 & 4.3 & 11.5 & 11 \\
\hline Rango & 5 & 5 & 3.7 & 9.1 & 7 \\
\hline Media & $5.17 \mathrm{a}$ & $4.89 \mathrm{~b}$ & $1.96 \mathrm{c}$ & $5.78 \mathrm{~d}$ & $8.15 \mathrm{c}$ \\
\hline Mediana & 6 & 5 & 1.8 & 5.4 & 8 \\
\hline Desviación estándar & 1.31 & 1.19 & 0.77 & 1.77 & 1.43 \\
\hline Coeficiente de variación (\%) & 25.24 & 24.25 & 39.31 & 30.64 & 17.6 \\
\hline \multicolumn{6}{|c|}{25 de noviembre } \\
\hline Estadística & NLP & NL7DAP & PHP & PHT & NLT \\
\hline Mínimo & 3 & 2 & 0.9 & 3.1 & 5 \\
\hline
\end{tabular}




\begin{tabular}{cccccc}
\hline Estadística & NLP & NL7DAP & PHP & PHT & NLT \\
\hline Máximo & 8 & 8 & 5.5 & 12.6 & 13 \\
Rango & 5 & 6 & 4.6 & 9.5 & 8 \\
Media & $5.16 \mathrm{a}$ & $5.33 \mathrm{a}$ & $2.03 \mathrm{c}$ & $6.37 \mathrm{c}$ & $9.08 \mathrm{a}$ \\
Mediana & 5.5 & 6 & 2 & 6.2 & 9 \\
Desviación estándar & 1.26 & 1.03 & 0.73 & 1.66 & 1.24 \\
Coeficiente de variación $(\%)$ & 24.39 & 19.33 & 36.27 & 26.12 & 13.63 \\
\hline
\end{tabular}

* = los promedios no seguidos por la misma letra en cada columna no eran significativamente diferentes entre sí utilizando la prueba $t$ para muestras independientes a un nivel de significación de $5 \%(p<0.05)$.

PHP y PHT fueron más altos el 22 de septiembre $(2.68 \mathrm{~cm}$ y $10.14 \mathrm{~cm}$, respectivamente). Los promedios de estos caracteres disminuyeron en fechas posteriores de siembra, lo que indica que la siembra temprana favoreció el crecimiento de los esquejes plantados el 9 de septiembre. A pesar del PHP más alto en septiembre, el valor de $2.68 \mathrm{~cm}$ fue significativamente menor que el recomendado para los esquejes plantados utilizando el método de multiplicación rápida (10$12 \mathrm{~cm})$.

NLT fue mayor en los esquejes plantados el 25 de noviembre, lo que sugiere que el desarrollo de plantas fue mayor en los tiempos posteriores de siembra. Las condiciones ambientales en fechas posteriores de siembra favorecieron el desarrollo de plantas de yuca. Estos resultados demostraron que la altura de la planta como criterio para la recolección de tallos puede presentar limitaciones porque el PHP varió entre las fechas de plantación y puede variar entre los cultivos. Además, el número de hojas representa adecuadamente la edad fisiológica de la planta (Streck et al., 2003).

Los promedios de BHMS fueron más altos en las plántulas trasplantadas el 9 de noviembre y el 3 de diciembre. No obstante, la SL fue mayor en las plántulas trasplantadas el 3 de diciembre (Cuadro 2). Este resultado puede haberse visto afectado por la altura de corte de los tallos en el momento de la cosecha. La altura de corte se estandarizó a 15-20 cm por encima de la superficie del suelo.

Tabla 2. Estadísticas descriptivas de las características de rendimiento de los tallos y raíces de las plántulas de yuca trasplantadas en cuatro fechas diferentes (9 de noviembre, 24 de noviembre, 3 de diciembre y 26 de diciembre).

\begin{tabular}{ccccccccccc}
\hline Estadística & BHMS & NB & NBS & SL & SD & RFW & RL & RD & NR \\
\hline & \multicolumn{7}{c}{9 de noviembre } \\
Min & 0.8 & 2 & 26 & 0.65 & 11.16 & 74.7 & 10.4 & 19.84 & 1 \\
Max & 2.12 & 4 & 52 & 1.9 & 29.31 & 3807 & 38.03 & 42.93 & 17 \\
Rango & 1.32 & 2 & 26 & 1.25 & 18.15 & 3732.3 & 27.63 & 23.09 & 16 \\
Media & $1.42^{*} \mathrm{a}$ & $2.94 \mathrm{~b}$ & $39.34 \mathrm{a}$ & $1.24 \mathrm{~b}$ & $21.26 \mathrm{a}$ & $1181.55 \mathrm{a}$ & $22.09 \mathrm{a}$ & $30.23 \mathrm{a}$ & $8.53 \mathrm{a}$ \\
Mediana & 1.4 & 3 & 39 & 1.22 & 21.43 & 997.1 & 21.6 & 29.67 & 9 \\
SD & 0.29 & 0.35 & 4.76 & 0.29 & 3.1 & 730.25 & 4.96 & 3.64 & 3.05 \\
CV $(\%)$ & 20.49 & 11.96 & 12.09 & 23.2 & 14.59 & 61.8 & 22.44 & 12.05 & 35.8 \\
\hline
\end{tabular}




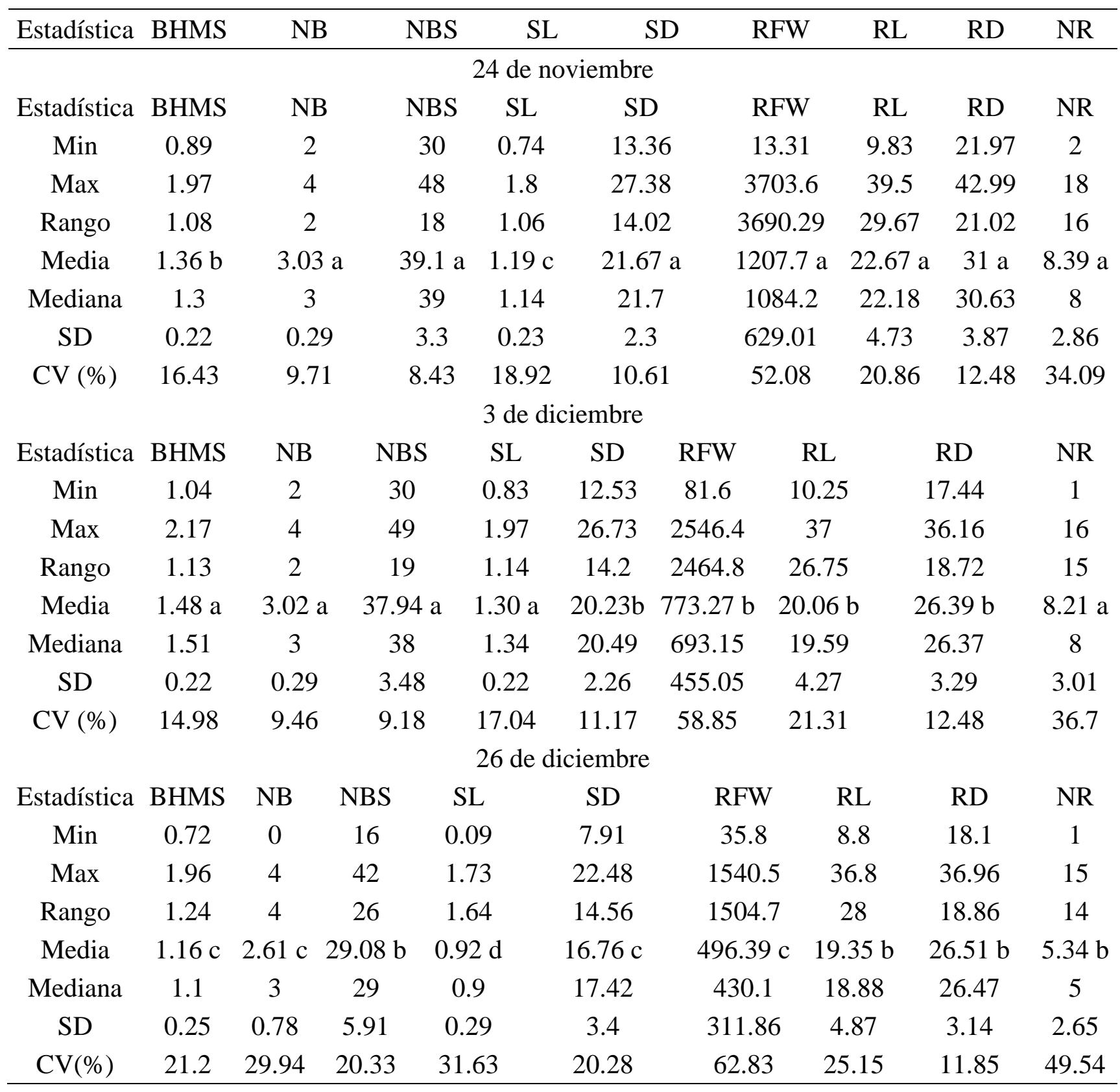

* = los promedios no seguidos de la misma letra en cada columna no eran significativamente diferentes entre sí utilizando la prueba $t$ para muestras independientes a un nivel de significación del $5 \%(p<0.05)$. Min= mínimo; Máx= máximo; $\mathrm{SD}=$ desviación estándar; $\mathrm{CV}=$ coeficiente de variación $(\%)$; $\mathrm{BHMS}=$ altura de ramificación del tallo principal $(\mathrm{cm}) ; \mathrm{NB}=$ número de ramas; $\mathrm{NBS}=$ número de brotes por tallo; $\mathrm{SL}=$ longitud del tallo $(\mathrm{cm}) ; \mathrm{SD}=$ diámetro del vástago $(\mathrm{mm}) ; \mathrm{RFW}=$ peso fresco de la raíz $\left(\mathrm{g}_{\text {planta }}{ }^{-1}\right) ; \mathrm{RL}=$ longitud de la raíz $(\mathrm{cm}) ; \mathrm{RD}=$ diámetro de la raíz $(\mathrm{mm}) ; \mathrm{NR}=$ número de raíces por planta.

El promedio de NB osciló entre 2.61 en plántulas trasplantadas el 26 de diciembre y 3.03 en plántulas trasplantadas el 24 de noviembre. El cultivo Apronta Mesa tiene tres ramas en el tallo principal. Este resultado indicó que NB se vio afectado por el momento del trasplante y que el trasplante tardío disminuyó los promedios de este rasgo. Del mismo modo, el promedio de NBS en la fecha del último trasplante fue de 29.08 . 
En las tres primeras fechas, el NBS osciló entre 37.94 y 39.34 y no difirió significativamente entre estas fechas, lo que indica que el trasplante tardío de plántulas limitó el desarrollo del tallo. Dado que el NBS es un parámetro relevante en el método de multiplicación rápida porque representa la cantidad de material propagativo cosechado para su uso en la próxima cosecha, el trasplante de plántulas en la tercera semana de diciembre limitó la producción de brotes por área unitaria.

La SD se redujo en las plántulas trasplantadas en diciembre. La mayor SD en las plantas trasplantadas en noviembre puede estar relacionada con el aumento del desarrollo de la zona de las hojas por la acumulación de sustancias de reserva en los tallos. Este rasgo mejora la calidad del tallo de yuca para su almacenamiento y selección de material propagativo para su uso en la próxima cosecha. La SD más alta es el resultado de la mayor cantidad de material de almacenamiento y agua, que son esenciales para el brote y el enraizamiento.

La médula tiene alta concentración de agua y está rodeada por la corteza, que tiene alto material de reserva. La SD y la relación entre la corteza y la médula afectan la capacidad de brotes y de enraizamiento de los esquejes después de la plantación. La RL fue mayor en las plántulas trasplantadas en noviembre y fue significativamente diferente a las plántulas trasplantadas en diciembre, con promedios de 22.09 y $22.67 \mathrm{~cm}$, con un mínimo de 9.83 y un máximo de $39.5 \mathrm{~cm}$.

No obstante, el rango en las cuatro fechas de trasplante osciló entre 26.75 y $29.67 \mathrm{~cm}$, lo que indica que hubo variabilidad en la RL y que el retraso en el trasplante redujo la RL media. Después de la definición morfológica de la cantidad de raíces de almacenamiento, hay un aumento significativo en RL (Figueiredo et al., 2014). En las tres primeras fechas de trasplante, el NR promedio osciló entre 8.21 y 8.53. En la última fecha de trasplante, NR fue de 5.34, que indica menor desarrollo del sistema radicular, especialmente las raíces de almacenamiento en plántulas trasplantadas más tarde.

La RFW fue mayor en las plántulas trasplantadas en noviembre y disminuyó con el retraso en el trasplante. Figueiredo et al. (2014) han demostrado que los parámetros de productividad de la raíz se definen en el siguiente orden: número, longitud, diámetro y peso fresco de las raíces. Por lo tanto, la reducción de NR puede afectar directamente a RFW, como se demostró en este estudio y la disminución se atenúa a medida que se retrasa el trasplante. Por lo tanto, NR es el factor limitante más significativo para el rendimiento de yuca en las temporadas tardías de trasplante de plántulas.

Se necesitaron tamaños de muestra más grandes (247 plantas) para estimar los promedios de rasgos con un rango de CI de $95 \%$ a $10 \%$ de la estimación promedio, es decir, cuando la precisión de estimación era mayor (Cuadro 3). Por el contrario, se requirieron 16 plantas para estimar promedios de todos los caracteres en todos los tiempos de siembra con un rango de CI de $95 \%$ a $40 \%$ (menor precisión). Por lo tanto, determinar el tamaño de la muestra en diferentes grados de precisión permite a los investigadores elegir el tamaño más adecuado para medir los rasgos agronómicos.

Se requirieron tamaños de muestra grandes para estimar los promedios de rasgos cuando la plantación se realizó el 7 de octubre, a excepción de PHP cuyo CV fue más alto (39.31\%) el 19 de octubre y se midieron 247, 63, 27 y 16 plantas para estimar promedios con rangos de CI de 95\%, $10 \%, 20 \%, 30 \%$ y $40 \%$, respectivamente. Este resultado demostró la relación entre el tamaño de la muestra y el CV, donde los CV fueron más altos para NLP, NL7DAP, PHP, PHT y NLT, el 7 de octubre, correspondientes al $28.87 \%, 33.61 \%, 37.34 \%, 37.5 \%$ y $19.14 \%$, respectivamente. Cargnelutti Filho et al. (2018a) observaron que se necesitaban tamaños de muestra grandes para estimar rasgos con CV más altos y el tamaño de la muestra era variable en las semillas de gandul (Cajanus cajan (L.) Millsp. 
Tabla 3. Tamaño de la muestra (número de plantas) con intervalos de confianza del $95 \%$ de rangos de $10 \%, 20 \%, 30 \%$ y $40 \%$ de las estimaciones promedio para el número de hojas en la siembra (NLP), número de hojas a los 7 días después de la siembra (NL7DAP), altura de la planta en la siembra (PHP), altura de la planta en trasplante (PHT), y número de hojas en trasplante (NLT) de plántulas de yuca en cuatro fechas de siembra ( 22 de septiembre, 7 de octubre, 19 de octubre y 25 de noviembre).

\begin{tabular}{|c|c|c|c|c|}
\hline & $10 \%$ & $20 \%$ & $30 \%$ & $40 \%$ \\
\hline \multicolumn{5}{|c|}{22 de septiembre } \\
\hline NLP & 106 & 25 & 11 & 6 \\
\hline NL7DAP & 173 & 41 & 18 & 11 \\
\hline PHP & 234 & 53 & 24 & 13 \\
\hline PHT & 220 & 54 & 23 & 14 \\
\hline NLT & 53 & 13 & 6 & 4 \\
\hline \multicolumn{5}{|c|}{7 de octubre } \\
\hline NLP & 138 & 33 & 13 & 9 \\
\hline NL7DAP & 182 & 45 & 19 & 11 \\
\hline PHP & 227 & 56 & 24 & 14 \\
\hline PHT & 220 & 54 & 24 & 15 \\
\hline NLT & 61 & 14 & 7 & 4 \\
\hline \multicolumn{5}{|c|}{19 de octubre } \\
\hline NLP & 100 & 27 & 11 & 6 \\
\hline NL7DAP & 94 & 22 & 11 & 6 \\
\hline PHP & 247 & 63 & 27 & 16 \\
\hline PHT & 145 & 35 & 17 & 9 \\
\hline NLT & 47 & 12 & 6 & 4 \\
\hline \multicolumn{5}{|c|}{25 de noviembre } \\
\hline NLP & 92 & 25 & 10 & 6 \\
\hline NL7DAP & 62 & 15 & 7 & 4 \\
\hline PHP & 208 & 52 & 22 & 13 \\
\hline PHT & 112 & 27 & 13 & 7 \\
\hline NLT & 29 & 8 & 3 & 2 \\
\hline
\end{tabular}

Los tamaños de muestra más pequeños eran necesarios para estimar los promedios de rasgos el 25 de noviembre y 10, 7, 22, 13 y 3 plantas eran necesarias para estimar NLP, NL7DAP, PHP, PHT y NLT, respectivamente, con un rango de CI de $95 \%$ a $30 \%$. Este resultado mostró que en esta fecha de plantación había menos variabilidad entre los caracteres medidos, lo que indica la mayor homogeneidad entre las plantas y, en consecuencia, la necesidad de utilizar tamaños de muestra más pequeños para estimar los promedios. Por el contrario, Bandeira et al. (2018a) constataron que la variabilidad de los datos en el cultivo de centeno (Secale cereale L. cv BRS Progresso) era mayor en la primera y última fecha de cultivo y en consecuencia, se requerían tamaños de muestra más grandes para estimar los promedios de los parámetros en esas fechas. 
Entre los rasgos analizados, se necesitaba un tamaño de muestra grande para estimar PHP y PHT en todas las fechas de plantación. Por el contrario, se necesitaron tamaños de muestra más pequeños para estimar rasgos discretos, especialmente NLT, correspondientes a 13, 14, 12 y 8 plantas el 22 de septiembre, 7 de octubre, 19 de octubre y 25 de noviembre, respectivamente, con un rango de CI de $95 \%$ a $20 \%$. Storck et al. (2007); Toebe et al. (2014) constataron que la variabilidad era mayor en los caracteres obtenidos mediante el pesaje en comparación con los obtenidos contando o midiendo en cultivos de maíz. La variabilidad en el tamaño de la muestra para estimar los promedios de rasgos en plántulas de yuca era baja, lo que permite al investigador elegir el tamaño de la muestra teniendo en cuenta sólo el conjunto de rasgos y el nivel deseado de precisión.

El trasplante realizado los días 9 y 26 de noviembre requirió mayores tamaños de muestra en comparación con el trasplante realizado el 24 de noviembre y el 3 de diciembre para medir personajes en diferentes niveles de precisión (Cuadro 4). Entre dos y 50 plantas debían medir los parámetros del sistema de brotes y raíces el 24 de noviembre, mientras que entre 3 y 68 plantas debían medir estos parámetros el 26 de diciembre con un rango de CI de $95 \%$ a 30\%. Bandeira et al. (2018b) constataron que había variabilidad en tamaño de muestra de acuerdo con los parámetros agronómicos analizados y las fechas de siembra en cultivos de centeno (Secale cereale L.).

Tabla 4. Tamaño de la muestra (número de plantas) con intervalos de confianza de $95 \%$ de $10 \%$, $20 \%, 30 \%$ y $40 \%$ de las estimaciones promedio para el rendimiento de tallo y raíz de plántulas de yuca en cuatro fechas de trasplante (11 de noviembre, 24 de noviembre, 3 de diciembre y 26 de diciembre).

\begin{tabular}{|c|c|c|c|c|}
\hline & $10 \%$ & $20 \%$ & $30 \%$ & $40 \%$ \\
\hline \multicolumn{5}{|c|}{11 de noviembre } \\
\hline BHMS & 65 & 16 & 8 & 5 \\
\hline NB & 24 & 6 & 3 & 2 \\
\hline NBS & 22 & 6 & 3 & 2 \\
\hline SL & 86 & 21 & 9 & 6 \\
\hline $\mathrm{SD}$ & 35 & 8 & 4 & 3 \\
\hline RFW & 617 & 153 & 70 & 40 \\
\hline RL & 84 & 20 & 8 & 5 \\
\hline $\mathrm{RD}$ & 23 & 6 & 3 & 2 \\
\hline NR & 206 & 50 & 24 & 13 \\
\hline \multicolumn{5}{|c|}{24 de noviembre } \\
\hline BHMS & 44 & 11 & 5 & 3 \\
\hline NB & 17 & 4 & 3 & 2 \\
\hline NBS & 13 & 3 & 2 & 2 \\
\hline SL & 57 & 14 & 6 & 4 \\
\hline $\mathrm{SD}$ & 17 & 5 & 2 & 2 \\
\hline RFW & 444 & 107 & 50 & 25 \\
\hline RL & 70 & 18 & 7 & 5 \\
\hline $\mathrm{RD}$ & 25 & 6 & 3 & 2 \\
\hline NR & 186 & 46 & 20 & 12 \\
\hline
\end{tabular}




\begin{tabular}{ccccc}
\hline & $10 \%$ & $20 \%$ & $30 \%$ & $40 \%$ \\
\hline BHMS & 37 & 3 de diciembre & 2 \\
NB & 17 & 9 & 4 & 2 \\
NBS & 14 & 4 & 3 & 2 \\
SL & 44 & 4 & 2 & 3 \\
SD & 20 & 11 & 6 & 2 \\
RFW & 545 & 5 & 2 & 33 \\
RL & 73 & 132 & 62 & 5 \\
RD & 25 & 18 & 8 & 2 \\
NR & 219 & 7 & 3 & 14 \\
& & 51 & 24 & 4 \\
BHMS & 70 & 18 & 8 & 7 \\
NB & 149 & 31 & 16 & 4 \\
NBS & 69 & 16 & 8 & 10 \\
SL & 163 & 38 & 20 & 4 \\
SD & 66 & 15 & 7 & 37 \\
RFW & 643 & 153 & 68 & 7 \\
RL & 100 & 26 & 11 & 23 \\
RD & 21 & 6 & 32 & 23 \\
NR & 402 & 103 & diciembre & \\
\hline
\end{tabular}

BHMS = altura de ramificación del tallo principal $(\mathrm{cm}) ; \mathrm{NB}=$ número de ramas; $\mathrm{NBS}=$ número de brotes por tallo; $\mathrm{SL}=$ longitud del vástago $(\mathrm{cm}) ; \mathrm{SD}=$ diámetro del vástago $(\mathrm{mm}) ; \mathrm{RFW}=$ peso fresco de la raíz $\left(\mathrm{g}\right.$ planta $\left.^{-1}\right) ; \mathrm{RL}=$ longitud de la raíz $(\mathrm{cm}) ; \mathrm{RD}=$ diámetro de la raíz $(\mathrm{mm}) ; \mathrm{NR}=$ número de raíces por planta.

Además, los autores observaron que las fechas intermedias de siembra requerían tamaños de muestra más pequeños, lo que indicaba una mayor homogeneidad de las plantas en estas fechas, similar al presente estudio. Estos resultados demostraron que había variabilidad entre los tiempos de siembra y subrayan la importancia de los estudios que utilizan diferentes tamaños de muestra y fechas de siembra para estimar los promedios de rasgos (Bandeira et al., 2018a).

Entre los parámetros del tallo, se necesitaban tamaños de muestra más grandes para estimar BHMS y SL en las cuatro fechas de trasplante. Por el contrario, se requirieron tamaños de muestra más pequeños para estimar NB y NBS, a excepción de NB en la última fecha de trasplante, que presentó un tamaño de muestra de 149 plantas, mientras que el tamaño fue de 24, 17 y 17 plantas el 9 de noviembre, el 24 de noviembre y de diciembre respectivamente, con un rango de CI de $95 \%$ a $10 \%$.

La mayor variabilidad en la emisión ramificada en las plantas cultivadas en la última fecha de trasplante requirió medir un mayor número de plantas para analizar este rasgo y se demuestra por un CV del $29.94 \%$ en la última fecha en comparación con 9.46-11.96\% en las otras fechas. Haesbaert et al. (2017) destacaron que se deben utilizar diferentes tamaños de muestra al mismo nivel de precisión para diferentes caracteres. Sin embargo, dadas las limitaciones de este método, el tamaño de muestra más grande debe utilizarse para estimar los promedios para tener en cuenta la variabilidad de rasgos. 
Con un rango de CI del 95\% del 20\%, el tamaño de la muestra para ST fue de 8, 5, 5 y 15 plantas el 9 de noviembre, el 24 de noviembre, el 3 de diciembre y el 26 de diciembre, respectivamente. Para esta característica y otras, el tamaño de la muestra se redujo o no se alteró desde el primero hasta la tercera fecha de trasplante. Esta tendencia no se mantuvo el 26 de diciembre y el número de plantas analizadas aumentó, a excepción de RD, que se mantuvo sin cambios en las cuatro fechas de los niveles de análisis de precisión.

En el caso de RD, el tamaño mínimo de la muestra era de tres plantas en cada fecha de trasplante con un rango de CI de $95 \%$ de $30 \%$. Sin embargo, el tamaño de la muestra varió entre rasgos en la misma fecha de trasplante a un cierto grado de precisión, como también observaron Toebe et al. (2014); Bandeira et al. (2018a). El CV para RFW osciló entre 52.08\% y 62.83\%, sugiere que es necesario un tamaño de muestra mayor para estimar RFW con un determinado nivel de precisión.

Estos resultados son similares a los de Cargnelutti Filho et al. (2018b), en el que el CV de parámetros de rendimiento en cultivo de lino (Linum usitatissimum L.) osciló entre $58.81 \%$ y 68.87\%. Cargnelutti Filho et al. (2018c) también encontró que la variabilidad en los rasgos de rendimiento era mayor que la de los rasgos morfológicos en los frijoles jack (Canavalia ensiformis). Cuanto mayor sea el CV presentado por un carácter, mayor será el tamaño de la muestra necesario para estimar los promedios (Toebe et al., 2014).

Independiente del tiempo de trasplante y el nivel seleccionado de precisión, la estimación de RFW requería tamaños de muestra grandes. La mayoría de los caracteres de raíz requerían tamaños de muestra grandes excepto para RD. Este se relacionó con el CV en RD, que osciló entre $11.85 \%$ y $12.48 \%$ en las cuatro fechas de trasplante. Cargnelutti Filho et al. (2018b) encontraron que los CV de rasgos morfológicos oscilaban entre $14.5 \%$ y $48.3 \%$, similar a los resultados actuales.

El alto número de plantas medidas y la variabilidad existente sugieren que el conjunto de datos analizado permitió calcular el tamaño de la muestra mediante remuestreo, como también observaron Cargnelutti Filho et al. (2018c); Bandeira et al. (2018a). A excepción de RFW, que requería tamaños de muestra grandes en cada fecha de trasplante con rango de CI de $95 \%$ de $20 \%$, midiendo 21 y 51 plantas es suficiente para estimar los caracteres de tallo y raíz, respectivamente.

Por lo tanto, si se mide los rasgos de estos dos grupos, el investigador puede elegir un tamaño mínimo de muestra de 51 plantas para trasplante entre el 9 de noviembre y el 3 de diciembre; es decir, en un experimento con cuatro repeticiones, 13 plantas necesitan ser muestreadas por repetición para estimar el promedio de un tratamiento. En el trasplante realizado el 26 de diciembre, el tamaño requerido de la muestra fue de 38 y 103 plantas para caracteres de brote y raíz, respectivamente, a excepción de RFW, que requirió 153, 107, 132 y 153 plantas el 9 de noviembre, el 24 de noviembre, el 3 de diciembre y el 26 de diciembre a un nivel de precisión de 20\%. Es evidente que la elección del grado de precisión depende del investigador sobre la variabilidad de los datos y la precisión deseada para estimar promedios de caracteres morfológicos y productivos en plantas de yuca propagadas utilizando el método de multiplicación rápida.

\section{Conclusiones}

Sesenta y tres plantas son suficientes para estimar promedios de rasgos de producción de plantas con un rango de CI de $95 \%$ al $20 \%$ y 153 plantas son suficientes para estimar promedios de rasgos de rendimiento de tallo y raíz con un rango de CI de $95 \%$ al $20 \%$. 


\section{Agradecimientos}

Al Consejo Nacional de Desarrollo Científico y Tecnológico $(\mathrm{CNPq})$ por la beca otorgada al primer autor.

\section{Literatura citada}

Bandeira, C. T.; Cargnelutti, F. A.; Carini, F.; Schabarum, D. E.; Kleinpaul, J. A. and Pezzini, R. V. 2018a. Sample sufficiency for estimation of the mean of rye traits at flowering stage. J. Agric. Sci. 10(3):178-186. https://doi.org/10.5539/jas.v10n3p178.

Bandeira, C. T.; Cargnelutti, F. A.; Follmann, D. N.; Bem, C. M.; Wartha, C. A. and Thomasi, R. M. 2018b. Sample size to estimate the mean of morphological traits of rye cultivars in sowing dates and evaluation times. Semina: Ciênc. Agrár. 39(2):521-532. http://dx.doi.org/10.5433/1679-0359.2018v39n2p521.

Brasil. 2009. O censo agropecuário 2006 e a agricultura familiar no Brasil. Brasília, MDA. 96 p.

Burin, C.; Cargnelutti, F. A.; Toebe, M.; Alves, B. M. and Fick, A. L. 2014. Dimensionamento amostral para a estimação da média e da mediana de caracteres de tremoço branco (Lupinus albus L.). Comum. Sci. 5(2):205-212. https://doi.org/10.14295/cs.v5i2.317.

Cargnelutti-Filho, A.; Alves, B. M.; Follmann, D. N.; Bem, C. M.; Kleinpaul, J. A.; Pezzini, R. V. and Simões, F. M. 2018a. Tamanho de amostra para a estimação da média de caracteres de sementes de feijão guandu anão. Agrarian. 11(41):294-298. https://doi.org/10.30612/ agrarian.v11i41.4577.

Cargnelutti-Filho, A.; Alves, B. M.; Santos, G. O.; Wartha, C. A.; Kleinpaul, J. A. and Silveira, D. L. 2018b. Sample size to estimate the mean and median of traits in flax. Revista Brasileira de Ciências Agrárias. 13(1):5492. https://doi.org/10.5039/agraria.v13i1a5492.

Cargnelutti-Filho, A.; Alves, B. M.; Toebe, M.; Burin, C. and Wartha, C. A. 2018c. Sample size to estimate the mean of traits in jack bean. Revista Brasileira de Ciências Agrárias. 13(1):1-7. https://doi.org/10.5039/agraria.v13i1a5505.

Comissão de química e fertilidade do solo. 2016. Manual de calagem e adubação para os estados do Rio Grande do Sul e de Santa Catarina. Sociedade Brasileira de Ciência do Solo, Núcleo Regional Sul. Comissão de Química e Fertilidade do Solo RS/SC. 376 p.

Egesi, C. N.; Ilona, P.; Ogbe, F. O.; Akoroda, M. and Dixon, A. 2007. Genetic variation and genotype $\times$ environment interaction for yield and other agronomic traits in cassava in Nigeria. Agron. J. 99(4):1137-1142. https://doi.org/10.2134/agronj2006.0291.

Figueiredo, P. G.; Bicudo, S. J.; Moraes-Dallaqua, M. A.; Tanamati, F. Y. and Aguiar, E. B. 2014. Componentes de produção e morfologia de raízes de mandioca sob diferentes preparos do solo. Bragantia. 73(4):357-364. http://dx.doi.org/10.1590/1678-4499.0150.

Haesbaert, F. M.; Lopes, S. J.; Mertz, L. M.; Lucio, A. D. and Huth, C. 2017. Tamanho de amostra para determinação da condutividade elétrica individual de sementes de girassol. Bragantia. 76(1):54-61. http://dx.doi.org/10.1590/1678-4499.389.

Hasibuan, S. and Nazir N. 2017. The development strategy of sustainable bioethanol industry on iconic Sumba island, Eastern Indonesia. Int. J. Adv. Sci. Eng. Inf. Technol. 7(1):276-283. http://dx.doi.org/10.18517/ijaseit.7.1.1796.

R-Development Core Team. 2014. R: a language and environment for statistical computing. Vienna, Áustria. 
Santos, V. S.; Souza, A. S.; Viana, A. E. S.; Ferreira-Filho, J. R.; Souza, K. A. S. and Menezes, M. C. 2009. Multiplicação rápida, método simples e de baixo custo na produção de material propagativo de mandioca. Boletim de Pesquisa e Desenvolvimento 44. Embrapa Mandioca e Fruticultura Tropical. 24 p.

Schoffel, A.; Lopes, S. J.; Koefender, J.; Lúcio, A. D.; Camera, J. N. and Golle, D. P. 2020. Sample size for estimation of averages of agronomic traits in cassava seedlings. Inter. J. Innovation Education Res. 8(5):73-82. https://doi.org/10.31686/ijier.vol8.iss5.2312.

Schons, A.; Streck, N. A.; Kraulich, B.; Pinheiro, D. G. and Zanon, A. J. 2007. Emissão de folhas e início de acumulação de amido em raízes de uma variedade de mandioca em função da época de plantio. Cienc. Rural. 37(6):1586-1592. http://dx.doi.org/10.1590/S010384782007000600013.

Silva, C. A. D.; Medeiros, E. V.; Bezerra, C. B.; Silva, W. M.; Barros, J. A. and Santos, U. J. 2013. Interferência da incorporação de matéria orgânica no solo no controle da podridão negra da mandioca, causada por Scytalidium lignicola. Biosci. J. 29(6):1823-1831.

Storck, L.; Garcia, D. C.; Lopes, S. J. and Estefanel, V. 2011. Experimentação vegetal. 3 (Ed.). UFSM. Santa Maria, UFSM. 198 p.

Storck, L.; Lopes, S. J.; Cargnelutti Filho, A.; Martini, L. F. D. and Carvalho, M. P. 2007. Sample size for single, double and three-way hybrid corn ear traits. Sci. Agr. 64(1):30-35. http://dx.doi.org/10.1590/S0103-90162007000100005.

Streck, N. A.; Weiss, A.; Xue, Q. and Stephen-Baenziger, P. 2003. Incorporating a chronology response into the prediction of leaf appearance rate in winter wheat. Ann. Bot. 92(2):181190. http://dx.doi.org/10.1093/aob/mcg121.

Toebe, M.; Cargnelutti-Filho, A.; Burin, C.; Casarotto, G. and Haesbaert, F. M. 2014. Tamanho de amostra para estimação da média e do coeficiente de variação em milho. Pesq. Agropecu. Bras. 49(11):860-871. http://dx.doi.org/10.1590/S0100-204X2014001100005.

Yuguda, R. M.; Girei, A. A.; Dire, B. and Salihu, M. 2013. Socio-economic factors and constraints influencing productivity among cassava farmers in Taraba State, Nigeria. Int. J. Adv. Agric. Sci. Technol. 1(1):1-15. 